\title{
Philosophy and Computing in Information Societies
}

\author{
Mariarosaria Taddeo ${ }^{1}$
}

Received: 17 August 2016/Accepted: 17 August 2016/Published online: 24 August 2016

(C) Springer Science+Business Media Dordrecht 2016

Philosophy and computing have often been related in the history of human culture. In the age of the information revolution, this relation has grown to define an entire area of philosophical enquiry. Over the past decades we have thought of this area as delineated by two Cartesian axes: conceptual and methodological. On the conceptual axis we find the deep philosophical questions concerning the concepts of information, computation, algorithms, cognition, intelligence, and language. On the methodological axis we find philosophical research developed through computer science methods. Formal methods and levels of abstraction are notable examples of these methodologies.

However, something is missing from this picture. As the information revolution unveils its full potential and our societies grow into mature information societies, we come to realise that computing has changed our daily practises and, more than that, it has provided us with new lenses to understand our environment and with new means to shape it - consider for example the impact of machine learning, Big Data, data science, and virtual reality on scientific research. These changes prompt new philosophical enquiries, which do not belong to the conceptual or the methodological axes, rather they are located on a third Cartesian axis, the contextual one.

Philosophical enquiries located along the contextual axis face new, pressing problems, concerning the way we produce scientific knowledge (philosophy of science), the nature of this knowledge (epistemology), and of the tools we use to produce it (philosophy of computing, philosophy of mathematics, and philosophy of statistics), as well as their ethical implications (ethics). It is with the contextual axis in the picture that the area of philosophy and computing is fully delineated and its importance becomes evident.

Mariarosaria Taddeo

mariarosaria.taddeo@oii.ox.ac.uk

1 Oxford Internet Institute, University of Oxford, Oxford, UK 
Philosophical analyses change over time to reflect their context: the scientific knowledge on which societies rely and the principles, both ethical and cultural, that shape them. The value of philosophy rests in this dynamism. Philosophy remains meaningful insofar as it changes to address the needs of the social, cultural, and historical context to which it belongs. A meaningful philosophy is a philosophy of its time. Together the three Cartesian axes ground philosophy in the information age. They embed it in its time and, in doing so, they contribute to enhance the value of philosophical analyses.

Since its foundation, Minds \& Machines has been a leading-and to a large extent a pioneering - journal offering a venue for publication of multidisciplinary research on philosophy and computing positioned on the conceptual or methodological axes. These remain central areas of enquiry, especially now as new milestones have been achieved in cognitive science, artificial intelligence, and quantum computing.

Nonetheless, I believe that the time has come to extend the scope of Minds \& Machines to philosophical enquiries located along the contextual axis. This is the vision that will guide my work as editor-in-chief. It rests on the conviction that in this way the journal will be able to serve a growing community of scholars interested in furthering our understanding of the reality in which we live and, by doing in so, in contributing to shape a better one for current and future generations.

To achieve this goal, Minds \& Machines will continue to publish the best research focusing on philosophy and computing, broadly understood. We will welcome high-quality articles, irrespective of their philosophical and disciplinary background and will assess them only with respect to the rigor of their approach and methodology. Many factors contribute to the success of a scientific journal, but publishing high-quality research remains the crucial one. For this reason, I hope that the research community served by Minds \& Machines will continue to find it an interesting and a valuable venue for publication.

Before leaving the reader to the third issue of 2016, I wish to express my gratitude to the authors and the reviewers who contributed to it. I am also very grateful to Gregory Wheeler, the former editor-in-chief of Minds and Machines and to the members of the scientific and executive boards. Their quality and enthusiastic commitment to the journal will be essential for it to continue to grow. I wish to express my deepest appreciation to Ties Nijssen, publishing editor at Springer; his help over the past months has been extraordinary and is only matched by the insightful conversations we have had on the relevance of philosophy and on the ways in which scientific journals can enhance it. 